\title{
Why a focus session on fetal ventriculomegaly and congenital hydrocephalus?
}

\author{
Soner Duru ${ }^{1}$ (DD
}

Received: 13 May 2020 / Accepted: 19 May 2020 / Published online: 4 June 2020

(C) Springer-Verlag GmbH Germany, part of Springer Nature 2020

\begin{abstract}
Introduction to the Special Focus Session on Fetal ventriculomegaly and Congenital Hydrocephalus.
\end{abstract}

Keywords Fetal ventriculomegaly · Congenital Hydrocephalus · Fetal Therapy

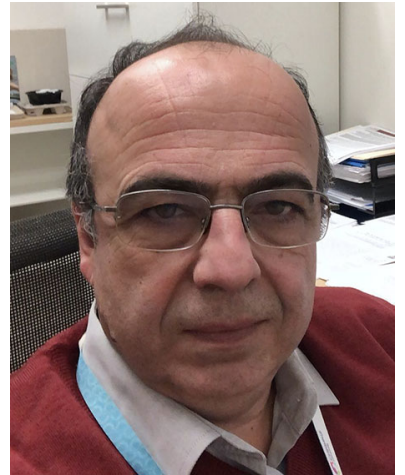

Fetal ventriculomegaly and congenital hydrocephalus are important global pathologic conditions. Pre- and postnatal management of pediatric hydrocephalus remains a challenge faced by clinicians and researchers.

Ventriculomegaly is characterized by dilation of the cerebral ventricles. The lateral ventricles are first seen by ultrasound at 13-14 weeks gestational age and change in morphology with subsequent development of the cerebral hemispheres. Fetal ventriculomegaly is diagnosed with prenatal ultrasound and, if necessary, magnetic resonance as an important diagnostic tool. Though overall ventricular volume increases with gestational age, the transverse atrial diameter remains relatively constant

Soner Duru

Soner.Duru@cchmc.org; sonerduru@yahoo.com

1 Center for Fetal and Placental Research, Center for Fetal, Cellular and Molecular Therapy, Division of Pediatric General and Thoracic Surgery, Cincinnati Children's Hospital Medical Center (CCHMC), 3333 Burnet Avenue, MLC 11025, Cincinnati, OH 45229-3039, USA during the second and third trimesters and is the preferred location for measurement. Fetal cerebral ventriculomegaly is defined as having atrial diameter of wider than $10 \mathrm{~mm}$ on prenatal ultrasound at any point in the gestation. The atrium of the lateral ventricle is located where the body, posterior horn, and temporal horn converge. Although mild, fetal ventriculomegaly is often incidental and benign. It can also be associated with genetic, structural, and neurocognitive disorders and outcomes ranging from normal to severe impairment. Hydrocephalus is one cause of ventriculomegaly and is defined as pathologic dilation of the cerebral ventricular system due to increased pressure, usually caused by obstruction.

In this special issue, expert authors examined the subject of fetal ventriculomegaly and congenital hydrocephalus as separate subtitles in terms of genetics, biophysics, neuropathological brain damage, neuroradiology, prenatal and postnatal follow-up, and treatment. According to the etiologic factors, additional genetic conditions and onset of hydrocephalus in the prenatal period, management, and treatment protocol may be changed.

In summary, this focus session pertaining to fetal ventriculomegaly and congenital hydrocephalus will provide updated knowledge about the spectrum of ventriculomegaly and hydrocephalus but also offer a review of the potential prenatal and currently surgical options available. We would like to again thank all of the contributors to this important focus session for their efforts and expertise in providing their up-to-date articles.

\section{Compliance with ethical standards}

Conflict of interest I have no conflict of interest to declare 